\title{
Automatic iterative evaluation of complex material constants in piezoelectric ceramics
}

\author{
C Alemany†, L Pardoł, B Jiménez $\nmid$, F Carmona $\nmid, \mathrm{J}$ Mendiola \\ and $A$ M Gonzálezł \\ † Instituto de Ciencia de Materiales (sede A) CSIC, Serrano 144, 28006 Madrid, \\ Spain \\ $\ddagger$ EUITT (UPM), Carr. de Valencia km 7, 28031 Madrid, Spain
}

Received 16 July 1993

\begin{abstract}
An automatic iterative method by which material constants of piezoelectric ceramics may be determined in complex form is described. Measurements are reported on samples with different shapes: thickness expansion and shear plates, and length expansion bars. With the constants provided by this method, the profiles for the conductance, resistance, susceptance and reactance are calculated, for the same sample, in the frequency range containing resonance and anti-resonance, and very good agreement is obtained with respect to the experimental data.
\end{abstract}

\section{Introduction}

The IEEE Standard on Piezoelectricity [1] describes well established methods to determine real, but not complex material constants. However, the increasingly extended use of lossy piezoelectric materials asks for alternative methods of measurement that provide information on the lag between an electrical (or acoustic) stimulus and the response of the material. This is supported, on the other hand, by the development of complex impedance spectrometry techniques, and by the computing capabilities of data acquisition equipment: the electric immitance is measured with precision in its real and imaginary components, and the possibility is now available for the relatively easy determination of elastic, dielectric and piezoelectric constants, from the general equations of piezoelectricity, as parameters with real and imaginary components. Furthermore, the ratio between these components provides, in the case of the elastic constants, the mechanical quality factor $Q$, which is of great practical interest but scarcely mentioned in the standards.

For the moment, it is generally accepted that, of the several methods proposed to obtain material constants in complex form [2-7], the best is that described by Smits [4] which uses an iterative method of calculation, because it provides results that are 'normally very accurate and useful in research work. However, it requires the judicious choice of three admittance or impedance data points, and this becomes tedious when a large number of samples need to be evaluated' [7].
To improve on that, an iterative and automatic method is described in this paper, for ceramic resonators in the form of thickness expansion plates, thickness shear plates and length expansion bars (both with field perpendicular and parallel to the bar's length). Smits' method is followed, but eliminating its constraints. Constants are determined in a frequency range containing the fundamental resonance mode and its corresponding anti-resonance. Profiles of $G$ (conductance), $R$ (resistance), $B$ (susceptance) and $X$ (reactance) are computed, and this leads to very good agreement with experimental data.

\section{Experimental method}

The piezoelectric ceramics for which results are reported here correspond to normal commercial materials (PZ34 (modified lead titanate) and PZ27 (lead zirconate titanate) manufactured by Ferroperm Ltd). Only the discs are obtainable from a catalogue; the rest of the shapes were machined by Ferroperm to comply with the requirements of the IEEE Standard and, thus, provide a reference with which to compare our results. These geometries were also poled by the manufacturer.

Samples were subject to sinusoidal electric stimuli in order to produce piezoelectric resonance, at room temperature, by means of an HP-4192A LF impedance analyser, controlled by a computer via a GPIB-PCIA, (National Instruments), interface board. The programs for data acquisition and further processing, in Microsoft 
QuickBASIC 4.5, are available from the authors without charge.

Frequency sweeps were carried out with the impedance analyser so as to cover only the interval in which fundamental resonance and anti-resonance take place, and measuring, at the same time, the two more adequate electrical parameters for each geometry. There are cases in which the analyser will display, and send to the computer, a saturated signal, whereas the measurement would be regular if it were made in another circuit mode. For instance, in the case of bar specimens with poling field applied parallel to the bar's length, it is preferable to measure $G$ and $B$ in the parallel circuit mode, instead of measuring $R$ and $X$ in the series mode, because this geometry involves high values of $R$ in antiresonance and the device saturates. The opposite applies to thickness expansion resonators. That is why, whatever the couple of electrical parameters being measured, in order to normalize for all geometries, we always stored in the computer and in data files the absolute electric admittance $|Y|$ and its phase angle $\theta$, and, from them, we generated the associated values of conductance $G=|Y| \cos (\theta)$, susceptance $B=|Y| \sin (\theta)$, resistance $R=\cos (\theta) /|Y|$, and reactance $X=-\sin (\theta) /|Y|$, both for the calculations and for the representation of the experimental resonance profiles.

From the position of the maxima of $G$ and $R$, the resonance $f_{\mathrm{s}}$ and anti-resonance $f_{\mathrm{p}}$ frequencies were inferred, and the corresponding electric immittance values stored. The two resonance bands were then swept with at least 20 experimental points so that, by means of a least-squares fit, it was possible to determine with precision the experimental half width $\Delta f_{s}$ of the resonance band (defined as the difference in frequencies between the minimum and the maximum of $B$ around $f_{\mathrm{s}}$ ) and the experimental half width $\Delta f_{\mathrm{p}}$ of the antiresonance band (difference in frequencies between the extremal values of $X$ around $f_{\mathrm{p}}$ ).

Apart from the frequency sweep, the computer demanded from the analyser, as many times as necessary during the iteration process, new measurements of $|Y|$ and $\theta$ for two other frequency values $f_{1}$ and $f_{2}$ (see below).

\section{Iteration procedure}

\subsection{Length expansion bar resonators with field perpendicular to their length}

The electrical admittance of a bar poled and excited in the direction of its thickness is obtained from the linear piezoelectric basic equations, and is given by the following expression in complex form

$$
\begin{aligned}
Y= & G+\mathrm{i} B=\mathrm{i} \frac{2 \pi f l w}{t}\left(\epsilon_{0} \epsilon_{33}^{\mathrm{T}}-\frac{d_{31}^{2}}{s_{11}^{\mathrm{E}}}\right) \\
& +\mathrm{i} \frac{2 w d_{31}^{2}}{t s_{11}^{\mathrm{E}} \sqrt{\rho s_{11}^{\mathrm{E}}}} \tan \left(\pi f l \sqrt{\rho s_{11}^{\mathrm{E}}}\right)
\end{aligned}
$$

where the density $\rho$, the length $l$, the width $w$, the thickness $t$, the universal constant $\epsilon_{0}$, and the driving frequency $f$ are real quantities. The other symbols refer to complex parameters, such as the piezoelectric charge coefficient $d_{31}$, the relative permittivity at constant stress $\epsilon_{33}^{\mathrm{T}}$ and the elastic compliance at constant $E, s_{11}^{\mathrm{E}}$. Other important parameters, obtained from the previous constants, are the coupling factor $k_{31}$, given by

$$
k_{31}^{2}=\frac{d_{31}^{2}}{\epsilon_{0} \epsilon_{33}^{\mathrm{T}} s_{11}^{\mathrm{E}}}
$$

and the elastic compliance at constant $D$

$$
s_{11}^{\mathrm{D}}=s_{11}^{\mathrm{E}}\left(1-k_{31}^{2}\right) \text {. }
$$

Except for the admittance $Y=G+\mathrm{i} B$, the rest of the complex quantities, both the set of the material constants and any combination between them, are written in the form

$$
\text { real part }-\mathrm{i} \times \text { imaginary part }
$$

and it is possible to assign a quality factor $Q$ to each parameter, defining it as the absolute value of the ratio real component/imaginary component. In this paper we shall only use $Q_{\mathrm{m}}$, the quality factor associated to the mechanical constant for each resonant mode, but making the distinction between parameters at constant $E$ and parameters at constant $D$.

The admittance $Y$ depends explicitly on the frequency of the measuring signal, but also implicitly because the three constants of the material are, in turn, a function of frequency. This dependence is not very strong and is usually neglected, to the extent that the relative permittivity at constant stress is normally measured at frequencies much lower than those used for the rest of the constants. However, one of the advantages of our method is that it makes it possible to determine all the constants within the same narrow margin of the frequency spectrum, covering the resonance and the anti-resonance of the fundamental mode, with the only simplification of ignoring its variation with frequency within the said interval.

Our aim being to determine, in complex form, the elastic constants (piezoelectric and dielectric) that appear in the previous formula for the admittance, this was done around the fundamental resonance by adopting, as a criterion of precision, the ability to reproduce in that interval the real and imaginary parts of both the admittance and the impedance bands of the experimental measurements by varying the frequency in equation (1).

The iteration process by which material constants have been determined is summarized in figure 1 .

Three measurements of $Y$, taken at three different frequencies around the fundamental resonance, should be mathematically sufficient to solve (by an iterative process because they are not linear equations) a system of equations of the type of equation (1), and thus obtain values for the three constants which are the unknowns of the system. However, in practice it is more convenient, as regards the automatization of the process, to use 


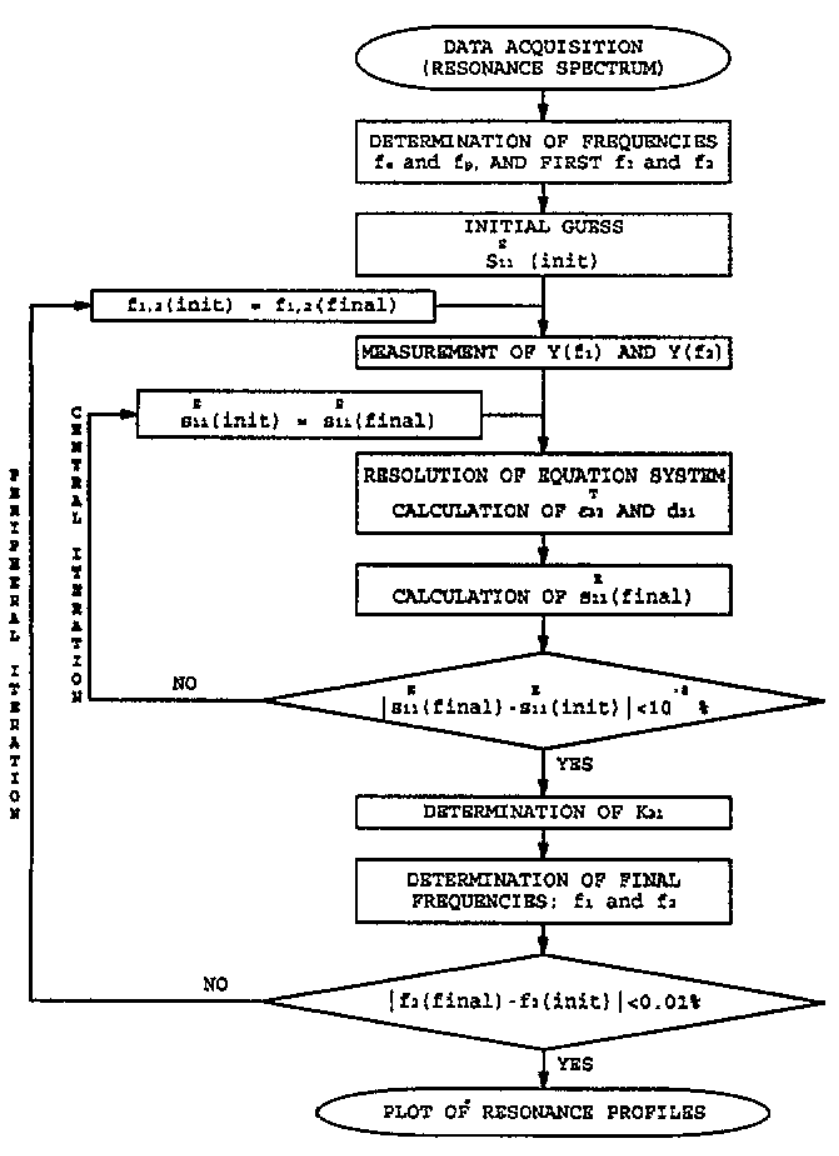

Figure 1. Block diagram of calculation process.

the experimental data from four measurements at four frequency values.

Two of the frequencies are determined, once and for all, in the data acquisition operation that takes place at the beginning of the process. These are the resonance frequency $f_{\mathrm{s}}$ corresponding to the maximum of the $G$ band, and the anti-resonance frequency $f_{\mathrm{p}}$ for which the maximum of the $R$ band occurs. They are generally accepted as most representative of the fundamental resonance of each piezoelectric mode, where the electric and elastic energies have extremal values.

The other two frequencies are obtained from the equation

$$
f_{1,2}=\frac{f_{s}}{\sqrt{1 \pm\left|k_{31}\right|}}
$$

defining the points with the maximum piezoelectric energy content [4]. The determination of these two frequencies is one of the two iterative processes involved in our method, and we shall call it peripheral iteration (see figure 1). But, the coupling factor $k_{31}$ being unknown, it is necessary to make an initial estimation of $f_{1}$ and $f_{2}$ so as to obtain four initial experimental values of $Y$ that will allow us to enter the central iteration loop in which $s_{11}^{\mathrm{E}}$ is determined. We shall take, as initial values for $f_{1}$ and $f_{2}$, the experimental values of the maximum and the minimum of $B$ around $f_{s}$, which correspond to half-height values in the resonance band:

$$
f_{2,1}=f_{\mathrm{s}} \pm \Delta f_{\mathrm{s}}
$$

For the central iteration we follow, almost ad pedem literae, the process described by Smits [4], adapting it to our election of frequencies. We thus begin by the same initial estimation for $s_{11}^{\mathrm{E}}$ :

$$
s_{11}^{E}=\frac{1}{4 \rho l^{2} f_{s}^{2}}\left[1-\mathrm{i}\left(\frac{\Delta f_{s}}{f_{s}}\right)\right] .
$$

With this value, and with the experimental values of $Y$ for frequencies $f_{1}$ and $f_{2}$, the piezoelectric charge coefficient $d_{31}$ and the relative permittivity at constant stress $\epsilon_{33}^{\mathrm{T}}$ are determined from a system of two linear equations with two unknowns.

We next calculate a better value of $d_{31}$, from the measurement of the admittance in $f_{\mathrm{p}}$ and from the rest of the parameters. This is the most important difference with respect to Smits' procedure, in which only the resonance band is considered.

Finally, from the experimental value of $Y$ in $f_{\mathrm{s}}$, a better approximation to the unknown $s_{11}^{\mathrm{E}}$ is obtained as a solution to an equation with an arctangent with a complex variable. The only precaution to be taken is that the angles with negative tangents belong to the second quadrant (and not to the fourth, which is the solution that computers routinely offer), because the fundamental resonance should correspond to angles next to $\pi / 2$.

A complete central iteration step has now been performed. If the modulus of the final value of $s_{11}^{E}$ differs from the initial one by more than $10^{-8} \%$, the central iteration process will be repeated as shown in figure 1. Only when the difference is lower will the central iteration loop be abandoned.

Two new values for frequencies $f_{1}$ and $f_{2}$ will now be calculated with equations (2) and (3), and for the peripheral iteration the cut-off criterion is established at a $0.01 \%$ difference between initial and final values of $f_{2}$. If the difference is higher the central iteration loop is entered once more, after measuring $Y$ at the new values of $f_{1}$ and $f_{2}$ that have just been tested, and considering as initial the last value of $s_{1 \mathrm{t}}^{\mathrm{E}}$.

The application of this method has shown that the initial and final values of $f_{1}$ and $f_{2}$ are quite different. On the other hand, we have observed that, from the very beginning, the coupling factor remains quite stable. We thus decided that the first central iteration should consist of just one step because this is sufficient to make a good estimation of $k_{31}$ which allows us to get (via equation (3)) into the peripheral iteration, which is repeated some three to five times before all the cut-off criteria are fulfilled and the evaluation may be considered as finished. The typical number of steps of the central iteration is from five to ten; the total number of steps therefore varies between 15 and 50 , and this amounts to the whole process, including intermediate measurements in $f_{1}$ and $f_{2}$, taking place in less than one minute.

\subsection{Length expansion resonators with field parallel to their length}

In this case it is more convenient to work with the impedance than with the admittance. The electrical 
impedance of a bar poled and excited along its length is given by

$$
\begin{aligned}
Z= & R+\mathrm{i} X=-\mathrm{i} \frac{l}{2 \pi f S}\left(\frac{1}{\epsilon_{0} \epsilon_{33}^{\mathrm{T}}}+\frac{g_{33}^{2}}{s_{33}^{\mathrm{D}}}\right) \\
& +\mathrm{i} \frac{g_{33}^{2}}{2 \pi^{2} S f^{2} s_{33}^{\mathrm{D}} \sqrt{\rho s_{33}^{\mathrm{D}}}} \tan \left(\pi f l \sqrt{\rho s_{33}^{\mathrm{D}}}\right)
\end{aligned}
$$

where the unknowns are the piezoelectric coefficient $g_{33}$, the relative permittivity at constant stress $\epsilon_{33}^{\mathrm{T}}$ and the elastic compliance at constant $D, s_{33}^{\mathrm{D}}$.

This geometry is the only one that permits, by piezoelectric resonance, determination of the piezoelectric charge coefficient $d_{33}$ as a function of the previous constants with the aid of $d_{33}=\epsilon_{0} \epsilon_{33}^{\mathrm{T}} g_{33}$. This is of great importance for the study of polarization processes because this coefficient is often related to the dipolar moment in ceramic samples.

The coupling factor $k_{33}$ is now given by:

$$
k_{33}^{2}=\left(1+\frac{s_{33}^{\mathrm{D}}}{\epsilon_{0} \epsilon_{33}^{\mathrm{T}} g_{33}^{2}}\right)^{-1}
$$

and the elastic compliance at constant $E$ by $s_{33}^{E}=$ $s_{33}^{\mathrm{D}} /\left(1-k_{33}^{2}\right)$.

As an initial estimation for the unknown $s_{33}^{D}$ we shall now take:

$$
s_{33}^{\mathrm{D}}=\frac{1}{4 \rho l^{2} f_{\mathrm{p}}^{2}}\left[1-\mathrm{i}\left(\frac{\Delta f_{\mathrm{p}}}{f_{\mathrm{p}}}\right)\right]
$$

whose real part is, in fact, the final value of the IEEE Standard.

By analogy with the previous geometry, in order to determine the frequencies $f_{1}$ and $f_{2}$ where measurements should be made, we use equation (3) adapted to this geometry, that is, substituting $k_{33}$ for $k_{31}$. However, it is the anti-resonance frequency $f_{\mathrm{p}}$ which appears in equation (7) and we have thus decided to take, as initial values for $f_{1}$ and $f_{2}$, the frequencies for the maximum and the minimum of $X$ around $f_{\mathrm{p}}$.

The two iterations take place in the same way and with the same cut-off criteria as in the previous mode.

\subsection{Thickness expansion plate resonators}

The more convenient equation in this case is

$$
\begin{aligned}
Z= & R+\mathrm{i} X=-\mathrm{i} \frac{t}{2 \pi f S \epsilon_{0} \epsilon_{33}^{\mathrm{S}}}+\mathrm{i} \frac{h_{33}^{2}}{2 \pi^{2} S f^{2} c_{33}^{\mathrm{D}} \sqrt{\rho / c_{33}^{\mathrm{D}}}} \\
& \times \tan \left(\pi f t \sqrt{\frac{\rho}{c_{33}^{\mathrm{D}}}}\right)
\end{aligned}
$$

where the unknowns are the piezoelectric coefficient $h_{33}$, the relative permittivity at constant strain $\epsilon_{33}^{\mathrm{s}}$ and the elastic stiffness at constant $D, c_{33}^{D}$.

We also use the coupling factor $k_{\mathrm{t}}$ given by:

$$
k_{\mathrm{t}}^{2}=\frac{\epsilon_{0} \epsilon_{33}^{\mathrm{S}} h_{33}^{2}}{c_{33}^{\mathrm{D}}}
$$

and the elastic stiffness $c_{33}^{\mathrm{E}}=c_{33}^{\mathrm{D}}\left(1-k_{\mathrm{t}}^{2}\right)$ and, as initial estimation for this quantity:

$$
c_{33}^{\mathrm{D}}=4 \rho t^{2} f_{\mathrm{p}}^{2}\left[1+\mathrm{i}\left(\frac{\Delta f_{\mathrm{p}}}{f_{p}}\right)\right]
$$

which is also the final value of the IEEE Standard.

Equation (3) is again used, but replacing the coupling factor by $k_{t}$ and taking the frequencies for the maximum and the minimum of $X$ around $f_{\mathrm{p}}$ as initial values for $f_{1}$ and $f_{2}$.

The rest of the characterization process is the same as before.

\subsection{Thickness shear plate resonators}

The behaviour in this mode can be described by exactly the same equations as for the thickness expansion samples, just by an adequate change of indexes. For piezoelectric ceramics, $h_{33}$ must be replaced by $h_{15}, \epsilon_{33}^{\mathrm{S}}$ by $\epsilon_{11}^{\mathrm{S}}, c_{33}^{\mathrm{D}}$ by $c_{55}^{\mathrm{D}}, c_{33}^{\mathrm{E}}$ by $c_{55}^{\mathrm{E}}$ and $k_{\mathrm{t}}$ by $k_{15}$.

\section{Results}

\subsection{Length expansion resonators with field perpendicular to their length}

Figures $2(a)$ and $2(b)$ show the experimental results for the fundamental resonance of a $2 \times 2 \times 20 \mathrm{~mm}^{3}$ length expansion resonator (PZ34 Ferroperm with $\rho=7.6 \mathrm{~g}$ $\mathrm{cm}^{-3}$ ) with field perpendicular. Also shown are the maxima of $G$ and $R$ (figure $2(a)$ ) and the spectra of $B$ and $X$ (figure $2(b)$ ), generated just by varying the frequency in equation (1) with the final values of the constants determined by the iterative method proposed in this work. These values of the constants are given in table 1, together with the results of the standard IEEE characterization of the same sample. From the calculated maxima of $G$ and $R$ and of the maxima and minima of $B$ and $X$, we obtain the quotient of the frequency of $G_{\max }$ to the difference between the frequencies of $B_{\max }$ and $B_{\min }$. A similar quotient is computed for the antiresonance band, just by substituting $R$ for $G$ and $X$ for $B$.

The piezoelectric resonance in these resonators is governed by equation (1), where the piezoelectric charge coefficient $d_{31}$ is squared. Therefore, from measurements of resonance to a sinusoidal electrical stimulus there will always be an indeterminacy as to the sign of this coefficient. The result of table 1 is thus inaccurate in this sense, but it must be pointed out that, whatever the sign, real and imaginary parts are of opposite sign.

\subsection{Length expansion resonators with field parallel to their length}

Experimental results and values calculated using equation (5) are represented in figures $3(a)$ (real parts of immitances) and $3(b)$ (imaginary parts) for the parallelepipedic sample of PZ27, with $\rho=7.7 \mathrm{~g} \mathrm{~cm}^{-3}, l=$ $20 \mathrm{~mm}$ and $S=4 \mathrm{~mm}^{2}$. Results are also summarized in table 2. 
Table 1. PZ34 length mode with field perpendicular.

\begin{tabular}{lccl}
\hline & Experimental & Standard & lterative \\
\hline$f_{s}(\mathrm{kHz})$ & 104.015 & & \\
$f_{\mathrm{p}}(\mathrm{kHz})$ & 104.045 & & \\
$C(\mathrm{pF}$ at $\mathrm{i} \mathrm{kHz})$ & 33.74 & & 55 \\
Number of iterations & & 0.0271 & $0.0274-0.0004 \mathrm{i}$ \\
$k_{31}$ & & 7.6011 & $7.6010-0.0074 \mathrm{i}$ \\
$s_{11}^{E}\left(\times 10^{-12} \mathrm{~m}^{2} \mathrm{~N}^{-1}\right)$ & & & 1031.20 \\
$Q_{\mathrm{m}}\left(s_{11}^{E}\right)$ & 1036.53 & & 1029.84 \\
$f_{\mathrm{s}} / \Delta f_{\mathrm{s}}$ & & 3.0701 & $3.0660-0.0539 \mathrm{i}$ \\
$d_{31}\left(\times 10^{-12} \mathrm{CN}^{-1}\right)$ & & 190.80 & $186.71-0.69 \mathrm{i}$ \\
$\epsilon_{33}^{\mathrm{T}}$ & & 7.5955 & $7.5953-0.0072 \mathrm{i}$ \\
$s_{11}^{\mathrm{D}}\left(\times 10^{-12} \mathrm{~m}^{2} \mathrm{~N}^{-1}\right)$ & & & 1056.01 \\
$Q_{\mathrm{m}}\left(s_{11}^{\mathrm{D}}\right)$ & & & 1050.97 \\
$f_{\mathrm{p}} / \Delta f_{\mathrm{p}}$ & 1055.79 & & \\
\hline
\end{tabular}

Table 2. PZ27 length mode with field parallel.

\begin{tabular}{lccl}
\hline & Experimental & Standard & Iterative \\
\hline$f_{\mathrm{s}}(\mathrm{kHz})$ & 71.0 & & \\
$f_{\mathrm{g}}(\mathrm{kHz})$ & 91.8 & & \\
$C(\mathrm{pF}$ at $1 \mathrm{kHz})$ & 3.02 & & 11 \\
Number of iterations & & 0.6720 & $0.6722-0.0028 \mathrm{i}$ \\
$k_{93}$ & & 9.6317 & $9.6265-0.0788 \mathrm{i}$ \\
$s_{33}^{\mathrm{D}}\left(\times 10^{-12} \mathrm{~m}^{2} \mathrm{~N}^{-1}\right)$ & & & 122.23 \\
$Q_{\mathrm{m}}\left(s_{33}^{\mathrm{D}}\right)$ & & & 122.40 \\
$f_{\mathrm{p}} / \Delta f_{\mathrm{p}}$ & 131.18 & 22.9198 & $23.6901-0.0470 \mathrm{i}$ \\
$g_{33}\left(\times 10^{-3} \mathrm{mVN}^{-1}\right)$ & & 1707.77 & $1598.92-31.03 \mathrm{i}$ \\
$\epsilon_{33}^{\mathrm{T}}$ & & 346.0897 & $334.9060-7.1643 \mathrm{i}$ \\
$d_{33}\left(\times 10^{-12} \mathrm{CN}^{-1}\right)$ & & 17.5640 & $17.5601-0.2642 \mathrm{i}$ \\
$s_{33}^{\mathrm{E}}\left(\times 10^{-12} \mathrm{~m}^{2} \mathrm{~N}^{-1}\right)$ & & & 66.46 \\
$Q_{\mathrm{m}}\left(s_{33}^{\mathrm{E}}\right)$ & & & 71.00 \\
$f_{\mathrm{s}} / \Delta f_{\mathrm{s}}$ & 71.30 & & \\
\hline
\end{tabular}

\subsection{Thickness expansion plate resonators}

Figures $4(a)$ and $4(b)$ show experimental results and calculated values of the fundamental resonance of a PZ34 Ferroperm disc resonator, with $\rho=7.6 \mathrm{~g} \mathrm{~cm}^{-3}$, $t=0.44 \mathrm{~mm}$ and $S=72.38 \mathrm{~mm}^{2}$. Table 3 summarizes the final results.

\subsection{Thickness shear plate resonators}

Finally, figures $5(a)$ and $5(b)$ show experimental and calculated results for the immitances in the fundamental resonance of a PZ27 Ferroperm thickness shear plate, with $\rho=7.7 \mathrm{~g} \mathrm{~cm}^{-3}, t=0.59 \mathrm{~mm}$ and $S=35 \mathrm{~mm}^{2}$. Table 4 summarizes the final results.

\section{Discussion}

\subsection{Iterative method}

The iterative process was repeated for all the samples changing the initial assumptions for the elastic constant, replacing $f_{s}$ by $f_{\mathrm{p}}$ in equation (4) (or $f_{\mathrm{p}}$ by $f_{\mathrm{s}}$ for other geometries) and by changing the sign of the imaginary part. Furthermore, for the determination of the initial frequencies $f_{1}$ and $f_{2}$, we interchanged bandwidths; and the replacement of $f_{s}$ by $f_{\mathrm{p}}$ (or vice versa) was also tested in equation (3). The major discrepancy observed was in the number of iterations, in some cases this increased slightly (as it should for a slower convergence). However, the final results, for the three principal parameters appearing in the fundamental equation of each resonance, are reproducible within 1 or $2 \%$, practically the same variation observed for different applications of the basic process. We thus feel that this 1 or $2 \%$ is rather the inaccuracy of the measurement method, due mainly to positioning of the samples, variations in ambient temperature, differences in calibration of the analyser within the frequency interval, etc. The error in the determination of secondary parameters will be obviously bigger due to accumulation. Given the complexity of the starting equations and the iterative processes used for solving them, we have not been able to carry out estimations, but we think, like Smits [5] that "errors due to indeterminacy of the dimensions, to spurious modes and to differences in the poling procedure are the most important ones, while those which can be attributed to the measurement procedure are of minor importance'.

Although characterization does not give appreciably different results when the above mentioned modifications are tested, frequencies $f_{1}$ and $f_{2}$ where measurements 

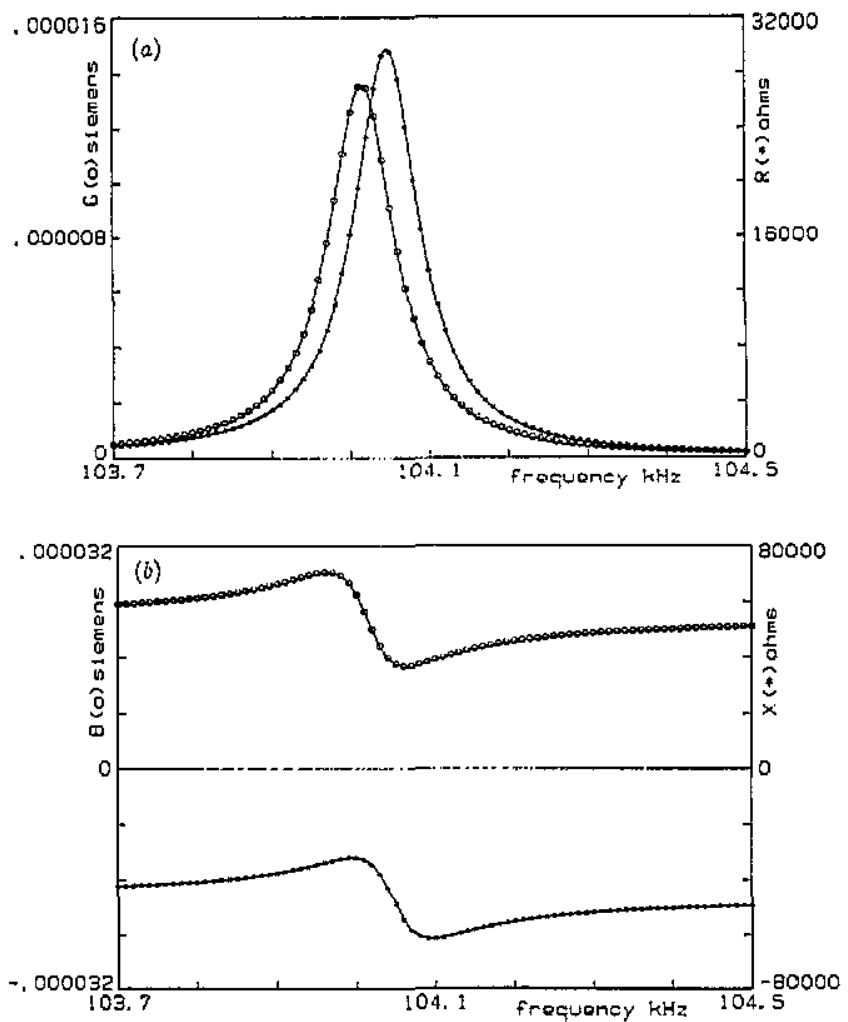

Figure 2. (a) Sample PZ34. Real parts of admittance $G$ and impedance $R$ for length mode with field perpendicular. (b) Sample PZ34. Imaginary parts of admittance $B$ and impedance $X$ for length mode with field perpendicular.
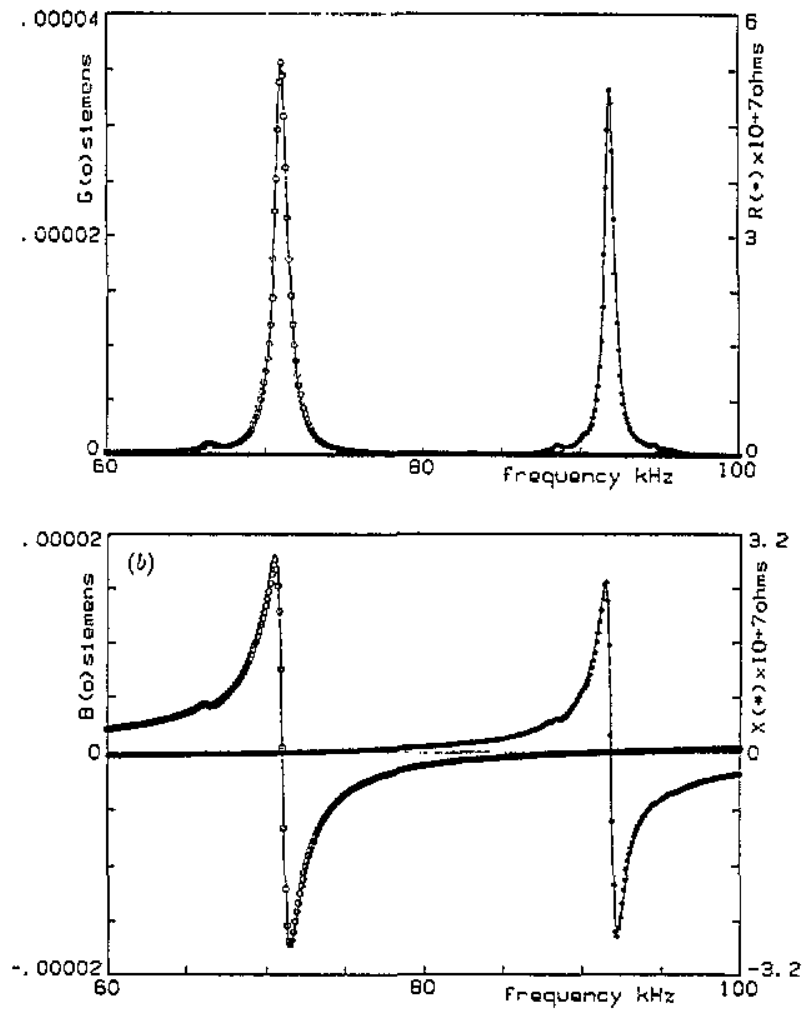

Figure 3. (a) Sample PZ27. Real parts of admittance $G$ and impedance $A$ for length mode with field parallel. (b) Sample PZ27. Imaginary parts of admittance $B$ and impedance $X$ for length mode with field parallel.

are made do show great changes when $f_{\mathrm{s}}$ is replaced by $f_{\mathrm{p}}$ (or vice versa). It looks as if these two
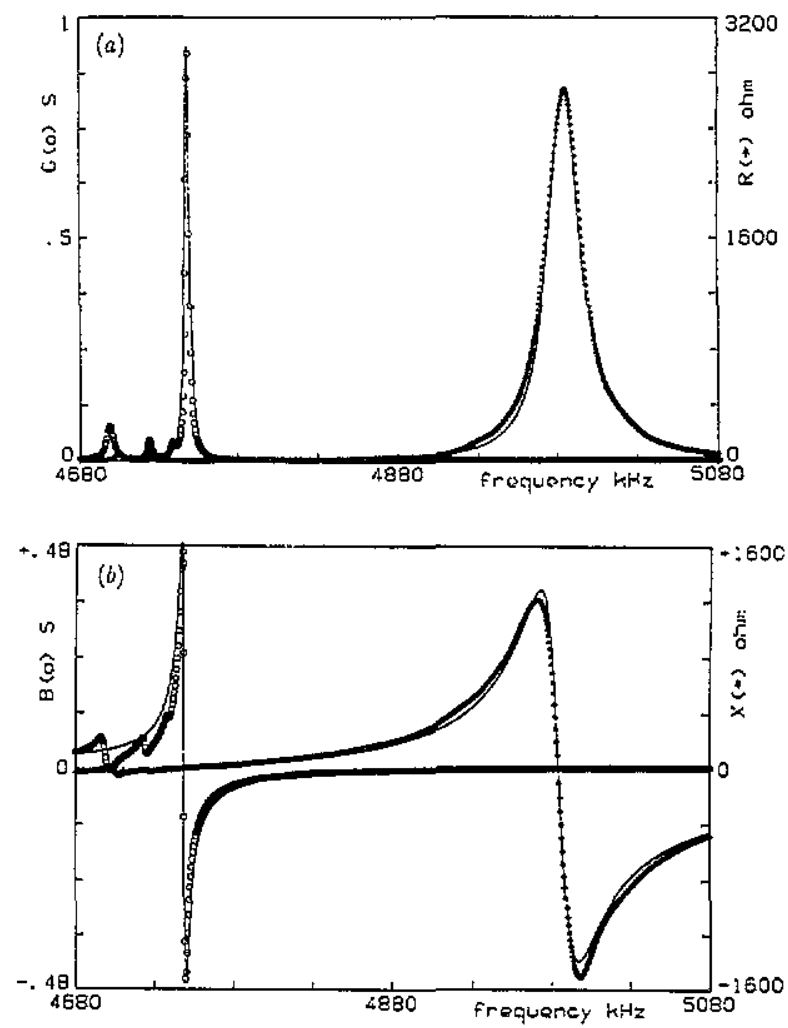

Figure 4. (a) Sample PZ34. Real parts of admittance $G$ and impedance $R$ for thickness mode of a disc. (b) Sample PZ34. Imaginary parts of admittance $B$ and impedance $X$ for thickness mode of a disc.
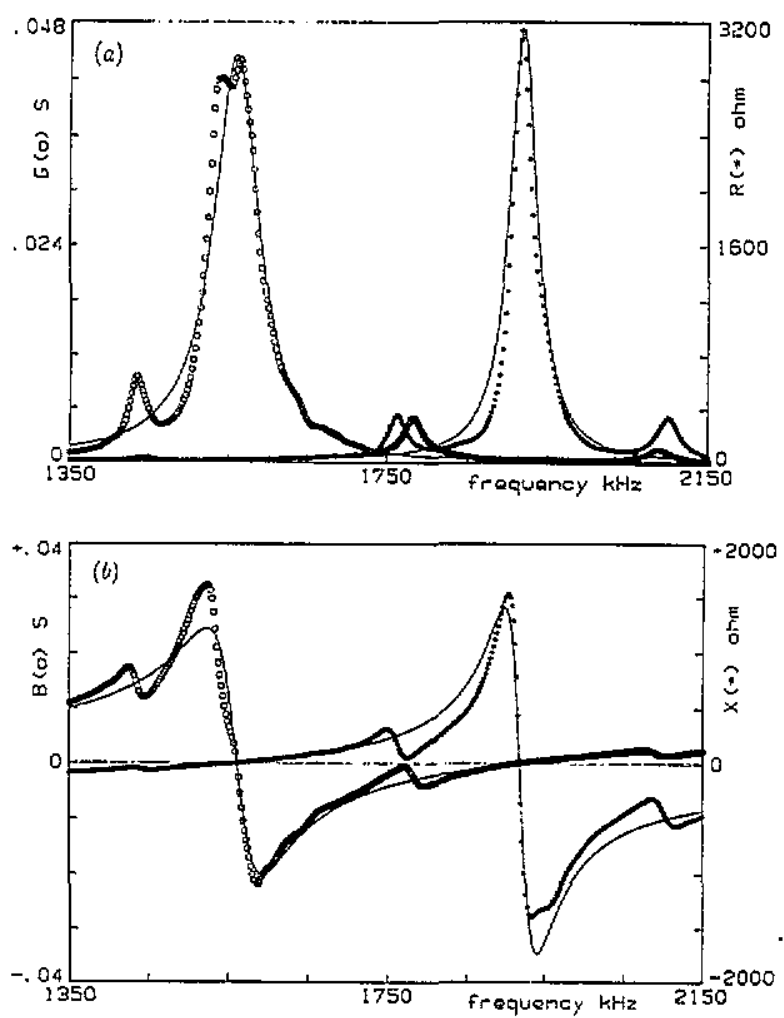

Figure 5. (a) Sample PZ27. Real parts of admittance $G$ and impedance $R$ for thickness shear mode of a plate (b) Sample PZ27. Imaginary parts of admittance $B$ and impedance $X$ for thickness shear mode of a plate.

experimental points were of minor importance, as compared with $f_{\mathrm{s}}$ and $f_{\mathrm{p}}$ which are determinant for 
Table 3. PZ34 disc.

\begin{tabular}{lccl}
\hline & Experimental & Standard & Iterative \\
\hline$f_{\mathrm{s}}(\mathrm{kHz})$ & 4748.5 & & \\
$f_{\mathrm{p}}(\mathrm{kHz})$ & 4984.5 & & \\
$C(\mathrm{pF}$ at $1 \mathrm{kHz})$ & 268.07 & & 30 \\
Number of iterations & & 0.3342 & $0.3338+0.0066 \mathrm{i}$ \\
$k_{\mathrm{t}}$ & & 14.6231 & $14.6190+0.0710 \mathrm{i}$ \\
$c_{\mathrm{B}}^{\mathrm{D}}\left(\times 10^{10} \mathrm{Nm}^{-2}\right)$ & & & 205.77 \\
$Q_{\mathrm{m}}\left(c_{33}^{\mathrm{D}}\right)$ & & & 203.45 \\
$f_{\mathrm{p}} / \Delta f_{\mathrm{p}}$ & 183.23 & 33.6101 & $34.7696+1.1074 \mathrm{i}$ \\
$h_{39}\left(\times 10^{8} \mathrm{Vm}^{-1}\right)$ & & $163.50^{*}$ & $152.27-2.92 \mathrm{i}$ \\
$\epsilon_{33}^{\mathrm{S}}$ & & 7.5955 & $12.9910-0.0014 \mathrm{i}$ \\
$c_{33}^{E}\left(\times 10^{10} \mathrm{Nm}^{-2}\right)$ & & & 9209.72 \\
$Q_{\mathrm{m}}\left(c_{33}^{E}\right)$ & & & 1356.71 \\
$f_{\mathrm{s}} / \Delta f_{s}$ & 1955.05 & & \\
\hline
\end{tabular}

* Determined using equation (158) of the IEEE Standard, after characterizing the radial mode and obtaining $k_{\mathrm{p}}=0.0370$.

Table 4. PZ27 thickness shear plate.

\begin{tabular}{lccl}
\hline & Experimental & Standard & Iterative \\
\hline$f_{\mathrm{s}}(\mathrm{kHz})$ & 1564 & & \\
$f_{\mathrm{p}}(\mathrm{kHz})$ & 1918 & & \\
$C(\mathrm{pF}$ at $1 \mathrm{kHz})$ & 707.2 & & 14 \\
Number of iterations & & 0.6182 & $0.6212-0.0131 \mathrm{i}$ \\
$k_{\mathrm{t}}$ & & 3.9441 & $3.9497+0.0875 \mathrm{i}$ \\
$c_{55}^{D}\left(\times 10^{10} \mathrm{Nm}^{-2}\right)$ & & & 45.13 \\
$Q_{\mathrm{m}}\left(c_{55}^{\mathrm{D}}\right)$ & & & 44.63 \\
$f_{\mathrm{p}} / \Delta f_{\mathrm{p}}$ & 63.81 & 14.3105 & $15.5841+0.1091 \mathrm{i}$ \\
$h_{15}\left(\times 10^{\mathrm{B}} \mathrm{Vm}^{-1}\right)$ & & $832.87^{*}$ & $709.71-24.02 \mathrm{i}$ \\
$\epsilon_{11}^{\mathrm{S}}$ & & 2.4370 & $2.4251+0.1178 \mathrm{i}$ \\
$c_{55}^{\mathrm{E}}\left(\times 10^{10} \mathrm{Nm}^{-2}\right)$ & & & 20.59 \\
$Q_{\mathrm{m}}\left(c_{55}^{\mathrm{E}}\right)$ & & & 22.29 \\
$f_{\mathrm{s}} / \Delta f_{\mathrm{s}}$ & 24.83 & & \\
\hline
\end{tabular}

* Calculated as iterative solution of equation (126) of the IEEE Standard.

the process, if a good reproducibility of the resonance spectra is asked for. They would merely be two points mathematically necessary in order to carry out the iteration, but whose automatic selection could have been performed according to another criterion, different from that which asks for points corresponding to a 'maximum piezoelectric energy content' [4]. Any other formula would do, around the fundamental resonance, as long as the possibility of repeated measurements is maintained. This has been checked by replacing the coupling factors by their squares in the selection of the said frequencies: the final results did not differ by more than $2 \%$.

\subsection{Applicability of the method}

The figures presented here are representative of the many cases and samples that have been studied, and it can be said that the reconstruction of the four profiles for the same sample is almost perfect both in the position and in the height of the peaks concerned.

The method is applicable even in cases in which the standard method is not, such as materials with a very small $k_{31}$ (values of $0.25 \%$ for this coupling factor have been measured in some $\mathrm{Ca}$-substituted lead titanates prepared in our laboratory).
In all the cases, the final result for the dielectric constant was a complex quantity with a negative imaginary part. This, given the general criterion adopted to represent material constants, amounts to real and imaginary parts having the same sign in the dielectric constant, as expected.

As a byproduct the method provides mechanical quality factors $Q_{\mathrm{m}}$ defined as the absolute values of the quotients between real and imaginary parts of the elastic constants. For each resonant mode there is an elastic constant which is directly implied in it, but there is another which may be immediately inferred: if the former was for $E$ constant, the latter will be for $D$ constant and vice versa. For each resonant mode we thus have two distinct mechanical quality factors for two distinct mechanical performances which correspond to two ways of using the material: short circuit and open circuit. On the other hand, for each resonant mode there are two bandwidths, around the resonance and anti-resonance peaks, although only one-resonanceis used to define the mechanical quality of a resonator, since Land et al published work [8] dealing with modes characterized by the admittance.

However, in this work we have seen how, when the fundamental equation of the resonance is governed 
by the impedance, that this implies that the elastic constant which is directly involved is for constant $D$, and the $Q_{\mathrm{m}}$ ascribed to this constant is very similar to the quotient $f / \Delta f$ of the anti-resonance band. We have also confirmed that, for the four geometries for which measurements are reported here, the $Q_{\mathrm{m}}$ values associated with the elastic parameters at constant $E$ correspond to $f_{\mathrm{s}} / \Delta f_{\mathrm{s}}$ in the resonance band, while $Q_{\mathrm{m}}$ associated to the parameters at constant $D$ may be assimilated to $f_{\mathrm{p}} / \Delta f_{\mathrm{p}}$ in the anti-resonance band.

\section{Conclusions}

The method described here has been successful in providing an accurate determination of the real and imaginary parts of material coefficients involved in the four modes of piezoelectric resonance of ceramic materials. The method consists of two iterations: one for the automatic selection of the frequencies in which complex electric immitances are to be measured, and another for solving nonlinear systems of equations in which complex piezoelectric constants are the unknowns.

The reconstruction of the resonance bands is a test that the method has passed with great success.

The determination of elastic constants in complex form permits one to define the mechanical quality factors (one for the resonance band and another for the antiresonance band) as ratios of real to imaginary parts of the elastic constants involved. These quality factors are related to the ratios $f / \Delta f$ of the corresponding peaks.

\section{Acknowledgments}

This work has been carried out under project MAT910422 of the Spanish CICYT. Thanks are given to Mrs W Wolny (Ferroperm Ltd, Kvistgård, Denmark) for supplying samples and also for machining them to adequate shapes and poling them.

\section{References}

[1] IEEE Standard on Piezoelectricity ANSI/LEEEStd 176-1987

[2] Holland R 1970 IEEE Trans. Sonic Ultrason. 17 123-4

[3] Xu Q C, Ramachandran A R and Newnham R E 1987 J. Wave-Mater, Interact. 2 105-22

[4] Smits J G 1976 IEEE Trans. Sonic Ultrason. 23 393-402

[5] Smits J G 1985 Ferroelectrics 64 275-91

[6] Sherrit S, Gauthier N, Wiederick H D and Mukherjee B K 1991 Ferroelectrics 119 17-32

[7] Sherrit S, Wiederick H D and Mukherjee B K 1992 Ferroelectrics 134 111-9

[8] Land C E, Smith G W and Westgate C R 1964 IEEE Trans. Sonics and Ultrason. 11 8-19 